\title{
Specifying a Model for Study of Safety Propaganda
}

\author{
Cruz García Lirios* \\ Academic Transdisciplinary Network, Mexico \\ *Corresponding Author: Cruz García Lirios, Academic Transdisciplinary Network, Mexico
}

\begin{abstract}
Background: Studies of propaganda, security, warn youth and old age; 1) the systematic dissemination of crimes attributed to political corruption; 2) state propaganda as legitimate security administrator his rectory; 3) the legitimation of citizens to consider them incapable of preventing crime initiatives; 4) older sectors are excluded by assuming that they are incapable of self - control and self - care.

Objective: Specify a model for studying the effects of propaganda security in the social representations of aging, youth and old age.
\end{abstract}

Method: A non-experimental, retrospective and exploratory study was conducted with a nonrandom selection of indexed sources the discretion of explanatory paths correlations between variables.

Results: The model included three hypotheses to explain the paths of correlations between four constructs and seven indicators for each.

Discussion: The revised theoretical, conceptual and empirical frameworks warn the inclusion of other variables such as helplessness, self-control, farsightedness, beliefs, attitudes and intentions that would complement the specified model.

Conclusion: A comprehensive model would explain the correlations paths from theoretical frameworks that establish the difference between crime prevention capabilities, systematic observation of corruption with emphasis on impunity.

Keywords: security, propaganda, social representations, attitudes, identity

\section{INTRODUCTION}

The aim of this study is to specify a model for the study of social representations of aging, youth and old age as a result of the propaganda of public safety.

For this purpose a non - experimental, retrospective and exploratory study was conducted with a nonrandom selection of indexed sources. The information was selected considering the conceptual dependency relationships between the explanatory variables of security and age. Subsequently, the information was processed into arrays of content analysis in order to establish the model. Then, the selected variables were included in the model from hypothesizing. Finally, he described the model was developed with the assumptions paths correlations.

\section{Propaganda Public Safety}

Propaganda, considered a security system that implements the state to legitimize his rectory in terms of crime prevention and the administration of justice, has been studied social sciences in general and psychology in particular. Therefore, propaganda has been disseminated in various media including television, radio, press and cinema had skewed the content and set the topics for discussion at a public agenda.

However, with the emergence of the Internet and social networks, state propaganda took on a new face to not only spread images and speeches, but now expressions of anger, fear, anger, surprise or anxiety that intensified helplessness and farsightedness of risk events and threats citizenship.

Thus, digital networks no longer build an agenda focused on issues but on terms that Internet users adopt to express their discontent or pleasure at any news, message, event or celebrity. 
However, traditional propaganda, unlike modern propaganda, generates reflections against and for the guidance of the State. This is because impacts differentially young audiences with respect to the largest public. While older adults used the radio and the press to have a close view of your local situation and the television and film to build a representation of the world in any case legitimated the rectory of the state and a nearby authority to represent him, young Netizens use networks to establish two types of threats focused on identity theft and ridicule.

Both aspects, the legitimation of state violence by the perception of insecurity and the feeling of helplessness, as well as the representation of the privacy and identity depend on digital networks, reveal state propaganda as omnipresent actor in society.

\subsection{Theory of Social Representations}

The theory of social representations is introduced by Serge Moscovici in 1961, from his doctoral thesis called Psychoanalysis his image and his public work addresses the social representation of psychoanalysis and the image of the psychoanalyst in modern French society to analyze the areas where social representations were configured. Moscovici takes as a basis the theoretical contributions of Durkheim collective representations which relate to the forms of thought that dominate a society, consisting of myths, religion, science, the dominant beliefs in a given society, which are incorporated in each one of its individuals (Piña, 2009).

This theory is recognized as a valuable contribution in the fields of social psychology, education, among others; now it constitutes an important reference for other social sciences for the study of social phenomena so the theory of social representations has been the theoretical foundation in conducting various investigations from various fields.

Moscovici addition, some of the main theorists of this theory are: Abric, Belisle, Flament, Farr, Guimelli, Ibanez, Heider, Jodelet, Mardellat, Mora, Pineapple, Shiele.

For Moscovici social representations are guides to action, determine behavior, social practices and dynamics of social relations. Cover retained information, selected and interpreted judgments made about the object and its environment, stereotypes and beliefs (Abric, 2001).

\subsection{According to Abric}

The representation functions as a system of interpretation of reality that governs the relations of individuals between their physical and social environment, and to determine their behavior or practices. It is a guide for action, guides the actions and social relations. It is a system of pre-decoding of reality since it determines a set of anticipations and expectations (Abric, 2001: 13).

For Moscovici representation it is always a representation of someone speaks and shows, communicates and expresses. After all it produces and determines behavior, because at the same time defines the nature of the stimuli around us and provoke us, and the meaning of the answers that we give them. The social representation is a particular form of knowledge, whose function is the development of behaviors and communication among individuals (Moscovici, 1961: 17).

The theory of social representations responds to four functions:

1. Functions know. They allow us to understand and explain reality. 2. Functions identity. Define the identity and allow safeguarding the specificity of the groups. 3. Functions Orientation: drive behaviors and practices and 4. Justice functions. Can justify a posteriori the postures and behaviors (Abric, 2001).

A social representation is composed of two elements. 1. The objectification regard to the development and operation 2. The anchoring of social representation. They can be analyzed in three dimensions: 1 . the information relates to the organization of the knowledge possessed by a group regarding a social object. 2. The field of representation or image, refers to the idea of image, social model, the specific and limited content of the proposals which relate to a specific aspect of the object of representation and 3. The attitude has to do with favorable or unfavorable in relation to the object of social representation (Piña, 2009) global orientation.

Enrique Pichon-Rivière raised "that man is a product in a very complex plot of ties and social relations; produced while determined, but at the same time producer, actor, protagonist. This 
complexity of relationships becomes tangible for the subject from its concrete conditions of existence or what is the same, from particular areas in and through which objectivity is constructed so that the conditions of society penetrate to the center itself individuality constructing a subjectivity permanently crossed by a particular social belonging ".

However, the "effect" on "the members of each society has a social reality that apparently share appears mediated by a complex network of links and meanings from which the" social "is emerging with a symbolic dimension that paralyzes any attempt mechanist to attribute a generic to a dialectical process of double construction "objectivity.

Subjectivity is understood as a socio - psychological construction that stands as the product of a permanent interpretation of the individual, the group and the social and projects in specific social contexts and ways of acting, thinking and feeling from which they are organized and They made tangible individualities that accompany tour of the human within its largest and most complex construction: society (Fuentes-Avila, 1995).

\subsection{Aging, Youth and Old Age}

If propaganda highlights the importance of identity and privacy while younger you are with respect to perceptions of insecurity of the elderly, then aging, youth and old age are transient categories set based on trust towards the contacts of the digital networks or in the case of the elderly, from their ability to represent their capabilities and resources, security, skills and knowledge.

Dementia is a syndrome characterized by a progressive deterioration of cognitive functions accompanied by psychiatric symptoms and behavioral disturbances resulting in progressive disability the patient (MJ Robles, 2011).

In Mexico City, the prevalence of dementia in people 65 and older is $4.7 \%$ and 3 to $6 \%$ in Latin America. (Gutierrez et al. , 2001; Mejía-Arango et al . , 2007). This figure can be anywhere from 20$30 \%$ when individuals are older (Mittelmark, 1994).

In Mexico there are programs designed by major public institutions such as the National Institute of Geriatrics, Care National Institute on Aging, Department of Health, etc., to serve the elderly, but from a very small picture, without offering an integrative approach, regardless of the patient, diagnosed with dementia, such as a human being who deserves respect and inclusion.

There is currently no curative treatment for people with dementia and care focuses on promoting wellness and improve the quality of life (Lucas-Carrasco, 2007).

Older adults are a vulnerable group to present a series of physical, biological and psychological characteristics that put them at greater disadvantage. The care of these children lies with the (mostly women) and to a lesser extent siblings, or by institutions, if the economic level allowed; and may be the case that they are abandoned.

In the Federal District, institutions have focused only provide seniors "certain" benefits that allow them to meet their basic needs, however, no specific public policies for the care of those diagnosed with $\mathrm{AD}$, offering them a Quality service. Also, there are no models for care that allows them to improve their quality of life.

Magdalena Contreras demarcation is one of the 16 delegations in the Federal District is divided. As governing bodies of the capital of the Republic they are decentralized delegations of the Federal District. It is located south west of Mexico City.

Of the 16 delegations Magdalena Contreras ranks ninth in extension, with a land area of 7458.43 hectares, accounting for $5.1 \%$ of total territorial Federal District. Of this area, 82.05\% (6,119.46 hectares) is ecological conservation area and the remaining $17.95 \%$ (1,338.97 hectares) is urban area

From this context it is necessary to carry out the study of social representations of the elderly in general and care of Alzheimer's in particular, since the media generate information that those involved in aging and care dementias assimilated as part of their cultural repertoire, which will define their strategies for treatment and prevention of mental illness.

Thus, the culture of respect for the elderly will determine family care as an inherent process to their family and groups of friends, but a culture that ponders youth over old age represents an abuse. 
Social representations are instances of processing information around which time is considered as a cultural process, but such is the influence of the group of belonging to the care of the elderly will be determined by the SCPs such as: "But know the devil that old devil "," as you see I saw, as I see I see "," If you want to grow old, then the tontejo haste ".

Therefore, the study of social representations from Alzheimer generate a comprehensive picture of the image of the caregiver against the attitudes of the elderly regarding the SCPs.

The diagnosis of social representations of Alzheimer care possible to anticipate scenarios of conflict between the elderly and the caregiver. In this sense, it will be feasible to explain the changes that are generated in the interplay between actors and propose models for intervention to reduce the effect of SCPs in the attitudes of the elderly and social representations of Alzheimer care.

\section{MODEL SPECIFICATION}

The model include three assumptions that explain the correlations between four constructs inferred by seven indicators set each as structural modeling criteria.

Hypothesis 1. The social representations of the guidance of the State security affect collective representations of solidarity and cooperation of the largest identities. The trajectory anticipated scenarios legitimization of violence of institutions committed to crime prevention and combating crime, even if the administration of justice is not guaranteed. The logical route explains the process in which older adults assimilate their dependence on the state and local authorities in order to establish law and order. Such trail warns that differences between youth identities and older identities involves two types of governance than in the case of older identities is only possible through the use of violence against those who violate the laws and in the case of youth identities They employ the ideological apparatuses such as the media.

Hypothesis 2. Social Security representations affect digital representations of youth identities privacy. It is a path that explains the influence of traditional media in digital networks, as disseminate information concerning the harassment (stalker, buller, troler) which would affect confidence towards the contacts on Facebook, Twitter, Instagram, WhatsApp Messenger or the main networks of interrelation among young identities.

Hypothesis 3. Although collective representations and digital representations are differentiated instruments of legitimation of State may be conjoined with the purpose of influencing any identity is juvenile or greater. This is the case of policies of common resources - information on environmental threats and risks, financial or social, that not only affect one sector or another, but could also affect future generations.

\section{DISCUSSION}

The contribution of this work to the state of knowledge lies in the specification of a model for the study of the incidence of social representations in collective representations, digital representations and youth identity or the identity of the elderly with regard to safety and privacy spread in the media.

However, from other theoretical and conceptual frameworks -auto control, agenda setting, elaboration likelihood, reasoned action, planners explanatory behavior trajectories dependency relationships between variables -indefensión, farsightedness, beliefs, attitudes are noted, intentions that could complement the specification of this work (Garcia and Carreon, 2013).

Some specified models have shown that the messages of the traditional media have more influence on audiences older than youth identities (García, 2012). This is because security policies are focused on the emotions of the audience and manage to penetrate their emotions more easily through television, as images and speeches are instruments of greater influence than the data in sectors with low educational and older (Garcia et al., 2013), although increasingly persuasion strategies are focused on the messages on local corruption administered by a federal entity (Garcia et al., 2015), generating a socio - political identity and no difference age groups, but processing capabilities (Garcia et al., 2014).

However, the study Mejia, Carreon and Garcia (2016) showed that older adults feel more guilt than youth with respect to insecurity, and they attribute to their age committing crimes against them. This means that the state propaganda directed to hold the non - citizen crime prevention, it affects mostly older adults who feel unable to confront the violence of civil courts. 
Therefore, a comprehensive model not only incorporate theoretical, conceptual and empirical frameworks, but also the perspective of young and older adults who watch propaganda differentially security, plus their perspective capacity generates an identity crime prevention but they may not always achieve that goal given the surrounding information regarding security at different levels and modalities.

\section{REFERENCES}

[1] Abric J. (1994). Social representations and practices. Mexico: Philosophy and Contemporary Culture.

[2] Carreon, J. and García, C. (2013). Theories of public safety and crime perception. Margen, 71: 1-16

[3] Fuentes, M. (1995). Subjectivity and social reality: A socio-psychological approach Journal of Psychology, 1, 107-120.

[4] Garcia, C. (2012). The structure of the perception of public insecurity. Liberabit, 18 (1), 37-44

[5] Garcia, C. Carreon, J. Hernandez, J. and Mendez, A. (2013). Systems sociopolitical violence. Polis, 36: 117

[6] Garcia, C. Carreon, J. Hernandez, J., Aguilar, J., Rosas, F. Morales, M. and Garcia, E. (2015). Reliability and validity of an instrument that measures perceptions of corruption.American Thought, 8) 15), 108-120

[7] Garcia, C. Carreon, J. Hernandez, J. Mejia, S., Garcia, and E. Rosas, F. (2014). Criminal social and political identity in Mexico. Hispano - American Notebooks Psychology, 14 (1) 5-16

[8] Lucas, R. (2007). Quality of life and dementia Medicine Clinic., 1, 349-354.

[9] Mejia, S. Carreon, J., Garcia, C. (2016).Psychological effects violence and insecurity. Eureka, 13 (1), 3955

[10] Moscovici, S. (1979). Psychoanalysis, its image and its public. Argentina: Huemul SA

[11] Rivera, B. Garcia, C. Garcia, E., Rosas, F., Lemon, G. Carreon, J. Hernandez, J. and Morales, M. (2015). . Contrast a model of political distrust Psiencia, 7, http://dx.doi.org/August1to1:1 0.5872/psiencia/7.1.21

[12] Robles, M. (2011). Diagnostic information in dementia. Spanish Journal of Geriatrics and Gerontology, 1, 163-169.

Citation: Cruz García Lirios. "Specifying a Model for Study of Safety Propaganda". International Journal of Research in Tourism and Hospitality (IJRTH), vol 5, no. 4, 2019, pp. 10-14. doi: http://dx.doi.org/10.204 31/2455-0043.0504003.

Copyright: () 2019 Authors. This is an open-access article distributed under the terms of the Creative Commons Attribution License, which permits unrestricted use, distribution, and reproduction in any medium, provided the original author and source are credited. 